\title{
Characterization of microRNAs and their target genes associated with transcriptomic changes in gamma-irradiated Arabidopsis
}

\author{
J.H. Kim ${ }^{1,2}$, Y.S. Go ${ }^{1}$, J.K. Kim ${ }^{1}$ and B.Y. Chung ${ }^{1}$ \\ ${ }^{1}$ Advanced Radiation Technology Institute, \\ Korea Atomic Energy Research Institute, Jeongeup-si, Jeollabuk-do, \\ Republic of Korea \\ ${ }^{2}$ Department of Radiation Biotechnology and Applied Radioisotope Science, \\ University of Science and Technology, Yuseong-gu, Daejeon, \\ Republic of Korea \\ Corresponding authors: J.H. Kim / B.Y. Chung \\ E-mail: jhongkim@kaeri.re.kr / chung@kaeri.re.kr
}

Genet. Mol. Res. 15 (3): gmr.15038386

Received January 6, 2016

Accepted February 19, 2016

Published July 29, 2016

DOI http://dx.doi.org/10.4238/gmr.15038386

Copyright (C) 2016 The Authors. This is an open-access article distributed under the terms of the Creative Commons Attribution ShareAlike (CC BY-SA) 4.0 License.

ABSTRACT. MicroRNAs (miRNAs) regulate gene expression in
response to biotic and abiotic stress in plants. We investigated gamma-
ray-responsive miRNAs in Arabidopsis wild-type and cmt3-11t mutant
plants using miRNA microarray analysis. miRNA expression was
differentiated between the wild-type and cmt3-11t mutants. miR164a,
miR169d, miR169h, miR172b*, and miR403 were identified as
repressible in the wild-type and/or cmt3-11t mutant in response to
gamma irradiation, while miR827, miR 840 , and miR850 were strongly
inducible. These eight miRNA genes contain UV-B-responsive cis-
elements, including G-box, I-box core, ARE, and/or MBS in the
putative promoter regions. Moreover, Box 4 , MBS, TCA-element, 
and Unnamed_4, as well as CAAT- and TATA-box, were identified in these eight miRNA genes. However, a positive correlation between the transcriptions of miRNAs and their putative target genes was only observed between miR169d and At1g30560 in the wild-type, and between miR827 and At1g70700 in the cmt3-11t mutant. Quantitative RT-PCR analysis confirmed that the transcription of miR164a, miR169d, miR169h, miR172b*, miR403, and miR827 differed after gamma irradiation depending on the genotype (wild-type, cmt3-11t, $d r m 2, d r d 1-6$, and $d d m 1-2)$ and developmental stage (14 or 28 days after sowing). In contrast, high transcriptional induction of miR840 and miR850 was observed in these six genotypes regardless of the developmental stage. Although the actual target genes and functions of miR840 and miR850 remain to be determined, our results indicate that these two miRNAs may be strongly induced and reproducible genetic markers in Arabidopsis plants exposed to gamma rays.

Key words: Arabidopsis thaliana; Gamma rays; Genetic marker; MicroRNA; Microarray; Transcriptome

\section{INTRODUCTION}

Small non-coding RNAs, which can be categorized into microRNAs (miRNAs) and small interfering RNAs (siRNAs), are important regulators of gene expression in biotic and abiotic stress responses in plants (Khraiwesh et al., 2012). Both miRNAs and siRNAs control gene expression at transcriptional and post-transcriptional levels, and are distinguished based on their biogenesis and function. Recently, the involvement of miRNAs in plant stress responses has been demonstrated in model plants under environmental stress conditions, including nutrient deficiency, drought, cold, salinity, bacterial infection, UV-B radiation, and mechanical stress (Khraiwesh et al., 2012; Kruszka et al., 2012). miR168, miR171, and miR396 commonly respond to high salinity, drought, and cold stress (Liu et al., 2008).

Ionizing radiation has been used commercially for mutation breeding of plants, for the prolonged storage of harvested commercial plants, and for plant quarantine services (Kim et al., 2013b). Ionizing radiation is a potent genotoxic agent and inducer of oxidative stress, which generates a wide range of genetic and epigenetic changes in the plant genome both directly, by energetic disruption of DNA integrity, and indirectly, through the formation of reactive oxygen species via water radiolysis (Esnault et al., 2010). Many of the miRNAs that are altered by ionizing radiation respond to other DNA-damaging agents, and are associated with the DNA damage response (Simone et al., 2009). Several miRNAs and their putative targets, such as miR24/miR-138 (H2AX), miR-182 (BRCA1), miR-421/miR-101 (ATM), and miR-504/miR-125b (p53), are known to be key regulators in the DNA damage response pathway (Joly-Tonetti and Lamartine, 2012). In contrast, many of the miRNA changes observed in response to ionizing radiation are also induced by $\mathrm{H}_{2} \mathrm{O}_{2}$ and are associated with reactive oxygen species or oxidative stress (Simone et al., 2009). Therefore, it is logical that many of the radiation-responsive miRNAs may play a role in the generalized cellular response to various oxidative stresses.

Since the study of miRNAs in response to ionizing radiation only began recently, the number of articles published in this research field is increasing exponentially. Strategies

Genetics and Molecular Research 15 (3): gmr.15038386 
used in these studies included the identification of putative targets of miRNAs involved in the DNA damage response, and the development of miRNA biomarkers of radiation exposure or radiosensitivity (Joly-Tonetti and Lamartine, 2012). The interactome of putative target genes of radiation-responsive miRNAs has facilitated the comprehensive modeling of the cellular stress response to ionizing radiation (Lhakhang and Chaudhry, 2012). However, since the response of miRNAs to ionizing radiation is limited in a cell type-, dose- and time-dependent manner, their use as indicators of radiation sensitivity rather than as useful biomarkers of radiation exposure, has been suggested (Joly-Tonetti and Lamartine, 2012).

Until recently, very few studies have been published on the identification of radiationresponsive miRNAs in plants. In relation to UV-B radiation, various miRNA gene families, including miR156, miR159, miR160, miR164, miR167, miR169, miR395, and miR171, were identified as UV-B radiation-responsive genes in Arabidopsis thaliana, Populus tremula, and/ or Triticum aestivum (Zhou et al., 2007; Jia et al., 2009; Wang et al., 2013). UV-B-responsive miRNA genes were found to possess various light-responsive and stress-related cis-elements in their upstream regions. In contrast, the only article to describe ionizing radiation and miRNAs in plants reported that miR164a, miR164c, miR164d, and miR156a-j were heavy ion-induced miRNAs in rice seedlings produced from germinated rice seeds exposed to heavy ion (Zhang et al., 2011a). Although highly conserved 5'-cis-element sequences have been investigated in gamma-ray-responsive Arabidopsis genes (Nagata et al., 2005), little is known about 5'-cis-elements in the putative promoter regions of plant miRNA genes responding to ionizing radiation. In addition, only a few miRNAs have been identified as regulators of DNA double-strand break repair during the DNA damage response (Joly-Tonetti and Lamartine, 2012); therefore, further study is needed to explore plant-specific miRNAs and their putative targets that respond to ionizing radiation-induced damage and discriminate between ionizing radiation and other environmental stresses. Therefore, our study aimed to identify gammaray-responsive miRNAs in Arabidopsis wild-type and cmt3-11t mutant plants after acute irradiation with gamma rays, in order to compare cis-elements in their putative promoter regions, and to identify their putative target genes via a plant small RNA target analysis server in association with genome-wide transcriptome data. The use of radiation-responsive miRNAs as widely applicable radiation markers or radiosensitivity indicators will also be discussed.

\section{MATERIAL AND METHODS}

\section{Plant materials, gamma-ray treatment, and RNA extraction}

Arabidopsis thaliana (ecotype Columbia wild-type and cmt3-11t mutant) plants were grown in soil or half-length MS medium under a 16 -h photoperiod at $22 / 18^{\circ} \mathrm{C}$ (day/night) for 28 days or at $23 / 20^{\circ} \mathrm{C}$ for 14 days, respectively. The cmt3-11t allele carries a T-DNA insertion in the predicted DNA methyltransferase domain of CMT3 predicted to create a null mutation (SALK_148381c). The wild-type and cmt3-11t T-DNA insertion mutant can be discriminated by DNA methylation and genome-wide transcriptome changes after exposure to gamma rays (Kim et al., 2013a).

Plants were irradiated with gamma rays at a dose of $50 \mathrm{~Gy} / \mathrm{h}$ for $4 \mathrm{~h}$ using a ${ }^{60} \mathrm{Co}$ gamma irradiator (IR-222, MDS Nordion Inc., Kanata, Canada) at the Advanced Radiation Technology Institute (ARTI). Next, plants were placed under growth conditions until 1 or 5 days after irradiation. For miRNA chip analysis, rosette leaves from at least 10 soil-grown plants

Genetics and Molecular Research 15 (3): gmr.15038386 
were pooled for use as individual biological replicates. In contrast, at least 10 medium-grown whole plants, including roots, were harvested for miRNA quantitative PCR (qPCR) analysis. All biological replicates were independently prepared from different batches of plants.

Total RNA was isolated using TRIzol reagent (Invitrogen, Carlsbad, CA, USA) for miRNA microarray and qRT-PCR analyses, while RNeasy ${ }^{\circledR}$ Plant Mini Kit (Qiagen, Chatsworth, CA, USA) was used for gene chip analysis. RNA integrity was assessed by analyzing the ribosomal RNA bands after gel electrophoresis or by using an Agilent 2100 Bioanalyser with an RNA 6000 Nano Kit (Agilent Technologies, Palo Alto, CA, USA).

\section{Microarray analysis}

\section{MicroRNA microarray experiments}

A total of 259 different miRNAs were identified from mature miRNA sequences for A. thaliana in the miRBase database (http://microrna.sanger.ac.uk/). Probes for each miRNA were designed using the Agilent eArray platform (http://earray.chem.agilent.com/earray/). Eight control probes were also designed as recommended by the eArray and used as negative controls. A total of 267 probes were synthesized and printed onto glass slides in an $8 \times 15-\mathrm{K}$ format by Agilent Technologies. For miRNA microarray analysis, biological triplicates of total RNA were isolated from three different batches of samples. Target RNA labeling and hybridization were performed using the Agilent miRNA Labeling Reagent and Hybridization Kit (Agilent Technologies) according to the manufacturer instructions. In brief, total RNA was subjected to dephosphorylation, denaturation, and labeling. Labeled RNA was hybridized with the Agilent miRNA microarray after desalting and denaturation. The hybridized microarrays were scanned using a DNA microarray scanner (Agilent Technologies) after washing. Scanned images were quantified by the Agilent Feature Extraction Software (Agilent Technologies).

\section{Gene chip experiments}

Genome-wide transcriptome analysis was performed with biological duplicates of total RNA from two different batches of samples using GeneChip ${ }^{\circledR}$ Arabidopsis ATH1 Genome Array (Affymetrix, Santa Clara, CA, USA). Experimental procedures have been previously described (Kim et al., 2013a). Raw data were registered as accession No. GSE43947 and GSM1074824-GSM1074839 in the NCBI Gene Expression Omnibus (GEO) database (http:// www.ncbi.nlm.nih.gov/geo/).

\section{Microarray data processing}

Raw data obtained from miRNA microarray or gene chip analyses were further processed by the GeneSpring GX 7.3 software (Agilent Technologies) as follows; i) probe signal values less than 0.01 were set to 0.01 , ii) per-chip normalization was set to the 50th percentile, and iii) each gene signal was normalized to the median value obtained in the experiment. Lists of differentially expressed genes (DEG) were obtained from probes with a $>2$-fold difference in the normalized signals between control and test samples. Gene ontology analysis of DEGs was performed via the web-based tool DAVID (Database for Annotation, Visualization, and Integrated Discovery, http://david.abcc.ncifcrf.gov/).

Genetics and Molecular Research 15 (3): gmr.15038386 


\section{miRNA qRT-PCR analysis}

Several gamma-ray-responsive miRNAs were selected from the miRNA microarray results and confirmed by quantitative RT-PCR analysis. Using Custom TaqMan ${ }^{\circledR}$ Small RNA Assay Kit (Applied Biosystems, Foster City, CA, USA), which includes ath-miR164a, ath-miR169d, ath-miR403, ath-miR840, or ath-miR850-specific primers, $5 \mathrm{~g}$ of each RNA sample was reverse-transcribed using TaqMan ${ }^{\circledR}$ MicroRNA Reverse Transcription Kit (Applied Biosystems) according to the manufacturer instructions. The resulting cDNA was amplified in 40 cycles on a quantitative thermal cycler, ABI 7300 (Applied Biosystems), using TaqMan $^{\circledR}$ Small RNA Assays and TaqMan ${ }^{\circledR}$ Universal PCR Master Mix II, No. UNG (Applied Biosystems) according to the manufacturer instructions.

\section{Cis-element analysis of miRNA genes and prediction of miRNA target genes}

To analyze putative promoter regions of gamma-ray-responsive miRNA genes, each premiRNA sequence obtained from miRBase was aligned with the $A$. thaliana genome sequence in the NCBI database. Approximately $1500 \mathrm{bp}$ of the upstream sequence of each miRNA gene was retrieved and used to identify cis-acting motifs in the putative promoter regions via PlantCARE (http://bioinformatics.psb.ugent.be/webtools/plantcare/) (Lescot et al., 2002).

Target genes of the gamma-ray-responsive miRNAs were predicted in psRNATarget, a plant small RNA target analysis server, using a preloaded $A$. thaliana transcript library (TAIR, version 10, cDNA, removed miRNA gene) released on 12/14/2010 for target search (Dai and Zhao, 2011). The default cut-off threshold of the maximum expectation was increased to 3.5 for higher prediction coverage.

\section{RESULTS AND DISCUSSION}

\section{microRNA microarray and gene chip experiments in gamma-irradiated Arabidopsis plants}

Biological effects of ionizing radiation, which acts as a genotoxic agent and oxidative stress inducer, are often compared with those of $\mathrm{H}_{2} \mathrm{O}_{2}$ or UV-B radiation (Nagata et al., 2005; Moon et al., 2008; Simone et al., 2009; Gill et al., 2015). For comparison, we performed a literature search to investigate what miRNAs respond to $\mathrm{H}_{2} \mathrm{O}_{2}$ or UV-B radiation as well as ionizing radiation (Table 1); however, only a few articles could be identified. Expression of the miR156 and miR164 families was found to respond to UV-B radiation and carbon ions (Jia et al., 2009; Zhang et al., 2011a; Casati, 2013; Wang et al., 2013), while expression of the miR169 family was affected by UV-B radiation and $\mathrm{H}_{2} \mathrm{O}_{2}$ (Zhou et al., 2007; Jia et al., 2009; Li et al., 2011). Previously, we demonstrated that genome-wide transcriptional changes in Arabidopsis seedlings subjected to gamma irradiation were most clear at $6 \mathrm{~h}$ and then decreased until $48 \mathrm{~h}$ (Kim et al., 2013b). The majority of transcriptomes responding to gamma rays differed from those responding to $\mathrm{H}_{2} \mathrm{O}_{2}$, especially at $24 \mathrm{~h}$. Therefore, in the present study, the putative association of gamma-ray-responsive miRNAs with gamma-ray-responsive transcriptomes was investigated using control and irradiated samples $24 \mathrm{~h}$ after gamma irradiation using miRNA microarray and gene chip experiments. In addition, since Arabidopsis wild-type and cmt3-11t mutants, which have a defective non-CG DNA methyltransferase, exhibited different 
genome-wide transcriptomes after gamma irradiation (Kim et al., 2013a), the gamma-rayresponsive miRNAs were compared between the two different genotypes of Arabidopsis.

\begin{tabular}{|c|c|c|c|}
\hline Stress type & Species & miRNA family & Reference \\
\hline UV-B & Arabidopsis thaliana & $\begin{array}{l}\text { miR156, miR 157, miR159, miR160, miR165, miR166, miR167, miR169, miR170, } \\
\text { miR171, miR172, miR393, miR398, miR401 }\end{array}$ & Zhou et al., 2007 \\
\hline UV-B & Populus tremula & $\begin{array}{l}\operatorname{miR} 156, \operatorname{miR} 160, \operatorname{miR} 164, \operatorname{miR} 165, \operatorname{miR} 166, \operatorname{miR} 167, \operatorname{miR} 168, \operatorname{miR} 398, \operatorname{miR} 408, \\
\text { miR } 159, \operatorname{miR} 169, \operatorname{miR} 390, \operatorname{miR} 393, \operatorname{miR} 395, \operatorname{miR} 399, \operatorname{miR} 472\end{array}$ & Jia et al., 2009 \\
\hline $\mathrm{H}_{2} \mathrm{O}_{2}$ & Oryza sativa & miR169, miR397, miR528, miR827, miR1425, miR319a.2, miR408-5p & Li et al., 2011 \\
\hline Carbon ion & Oryza sativa & miR156, miR164, miR2097-5p & Zhang et al., 2011a \\
\hline UV-B & Zea mays & $\begin{array}{l}\text { miR156, miR164, miR 165, miR166, miR171, miR172, miR395, miR396, miR398, } \\
\text { miR399, miR444, miR529, miR533a*, miR896, miR903, miR1427, miR1858a }\end{array}$ & Casati, 2013 \\
\hline UV-B & Triticum aestivum & miR156, miR159, miR164, miR167, miR171, miR395 & Wang et al., 2013 \\
\hline
\end{tabular}

\section{Identification of gamma-ray-responsive miRNAs in Arabidopsis wild-type and cmt3-11t mutant plants}

The overall expression pattern of the gamma-ray-responsive miRNAs differed by time after gamma irradiation and by the genotype of genome-wide DNA methylation (Table 2). The levels of miR164a, miR172b*, and miR403 expression 1 day after gamma irradiation significantly decreased in the cmt3-11t mutant, but not in the wild-type, while the level of miR827 increased only in the wild-type. In particular, transcriptional changes of miR164a, miR169d, and miR403 followed an opposite trend in the cmt3-11t mutant compared to the wild-type in response to gamma rays. This difference can be explained in part by the pronounced differences in genome-wide gamma-ray-responsive transcriptome profiles between the wild-type and cmt3$11 t$ mutant (Kim et al., 2013a). The gamma-ray-responsive miRNAs seem to be significantly affected by the genome-wide DNA methylation level. Expression of the miR164 family has also been reported to be induced by UV-B radiation and carbon ion (Jia et al., 2009; Zhang et al., 2011a) and expression of miR169 and miR827 was substantially increased after $\mathrm{H}_{2} \mathrm{O}_{2}$ treatment (Li et al., 2011). In the present study, these miRNAs may be associated with general light- or stress-related signals induced by gamma irradiation. In contrast, the expression levels of miR840 and miR850 were remarkably increased in both the wild-type and cmt3-11t mutant 1 day after gamma irradiation. There are no reports to date on such transcriptional induction of miR840 and miR850 in response to environmental stress factors. This indicates that these two miRNAs are highly specific to gamma rays and may be used as radiation markers for the early radiation

\begin{tabular}{|c|c|c|c|c|c|c|}
\hline \multirow[t]{2}{*}{ miRNA } & \multirow[t]{2}{*}{ miRBase accession } & \multirow[t]{2}{*}{ miRBase sequence } & \multicolumn{2}{|c|}{ Wild-type } & \multicolumn{2}{|c|}{$\mathrm{cmt3-11t}$} \\
\hline & & & 1 day & 5 days & 1 day & 5 days \\
\hline miR164a & MIMAT0000185 & UGGAGAAGCAGGGCACGUGCA & $>2.0,1$ of $3^{\dagger}$ & & $0.011 \pm 0.000$ & \\
\hline $\operatorname{miR} 169 \mathrm{~d}$ & MIMAT0000908 & UGAGCCAAGGAUGACUUGCCG & $>2.0,1$ of $3^{\dagger}$ & $0.196 \pm 0.264$ & $<0.5,1$ of $3^{\dagger}$ & $0.160 \pm 0.259$ \\
\hline miR169h & MIMAT0000913 & UAGCCAAGGAUGACUUGCCUG & & $0.297 \pm 0.107$ & & $0.380 \pm 0.150$ \\
\hline miR172b* & MIMAT0000204 & GCAGCACCAUUAAGAUUCAC & & & $0.362 \pm 0.024$ & \\
\hline miR403 & MIMAT0001004 & UUAGAUUCACGCACAAACUCG & $>2.0,1$ of $3^{\dagger}$ & & $0.011 \pm 0.001$ & \\
\hline $\operatorname{miR} 827$ & MIMAT0004243 & UUAGAUGACCAUCAACAAACU & $138.184 \pm 21.127$ & & & \\
\hline $\operatorname{miR} 840$ & MIMAT0004262 & ACACUGAAGGACCUAAACUAAC & $181.913 \pm 11.394$ & $194.855 \pm 6.120$ & $181.396 \pm 28.143$ & \\
\hline miR850 & MIMAT0004272 & UAAGAUCCGGACUACAACAAAG & $166.951 \pm 33.511$ & & $150.423 \pm 27.080$ & \\
\hline
\end{tabular}

Eight miRNAs in the list showing a $>2$-fold change in expression at 1 and/or 5 days after gamma irradiation in 2-3 independent experiments. Values represent the mean fold-change and standard deviation of 2-3 independent experiments. ${ }^{\dagger} 2$-fold change in one of three independent experiments. 
response 1 day after gamma irradiation. In addition, since the levels of miR169d and miR169h expression were substantially reduced in both plants 5 days after gamma irradiation, the miR169 family may also be a radiation marker for the late radiation response in Arabidopsis plants.

\section{Prediction of cis-elements in the upstream regions of gamma-ray-responsive miRNA genes}

Distinctive cis-elements in the 5 -upstream sequences or putative promoter regions of UV-B-responsive miRNA genes have been identified in A. thaliana, Populus tremula, and Triticum aestivum (Zhou et al., 2007; Jia et al., 2009; Wang et al., 2013). Many of the UV-Bresponsive cis-elements including G-box, I-box core, ARE, and MBS were frequently found in the putative promoter regions of gamma-ray-responsive miRNA genes (Table 3). However, GT-1 sites were relatively rare in the gamma-ray-responsive miRNA genes, while Box 4 and TCA-elements were found preferentially in these genes. Light relevant G-box and I-box core, and stress-related ARE and MBS may be important cis-elements for the transcriptional regulation of miRNAs responding to both UV-B radiation and gamma rays. Instead, stressrelated TCACG-motif and TCA-element seem to be associated with the expression of gammaray-responsive miRNA genes. Additionally, all the miRNA genes listed included a large number of CAAT- and TATA-boxes, which are generally found in the 5'-upstream regions of most eukaryotic genes (Table 4). As well as Box 4, G box, GA motif, MBS, and TCA-elements, CGTCA-motif, Skn-1 motif, and Unnamed 4 were identified as common cis-elements in the highly induced miRNAs, (e.g., miR827, miR840, and miR850), responding to gamma rays. Although many of the miRNA genes responding to gamma rays and UV-B radiation showed similar levels of $c i s$-elements in their putative promoter regions, our results suggest that the possession of specific cis-elements, novel gamma-ray-specific cis-elements, or other

Table 3. Major predicted light-related and stress-responsive cis-elements in the putative promoter regions of gamma-ray-responsive miRNA genes.

\begin{tabular}{|c|c|c|c|c|c|c|c|c|c|}
\hline & miR164a & $\operatorname{miR} 169 \mathrm{~d}$ & $\mathrm{miR} 169 \mathrm{~h}$ & miR172b* & $\mathrm{miR} 403$ & $\operatorname{miR} 827$ & $\operatorname{miR} 840$ & $\operatorname{miR} 850$ & Total \\
\hline 3-AF1 binding site (AAGAGA) & 1 & & & 1 & & & & 1 & 3 \\
\hline AE-box (AGAAACTT) & & & 1 & 1 & & 1 & & & 3 \\
\hline Box 4 (ATTAAT) & 8 & 2 & 1 & 4 & 5 & 1 & 1 & 1 & 23 \\
\hline Box I (TTTCAAA) & 1 & 1 & 1 & & & & 2 & 5 & 10 \\
\hline Box II (TGGTAATAA) & 1 & & & & 1 & & 1 & & 3 \\
\hline CATT-motif & & & 1 & 1 & & & 1 & & 3 \\
\hline G-box (CACGTG) & 6 & & & 1 & 4 & 1 & 2 & 8 & 22 \\
\hline GA-motif & & 1 & 1 & & & 1 & 1 & 1 & 5 \\
\hline GAG-motif & 2 & & 3 & & & 4 & & 2 & 11 \\
\hline GT-1 site (GGTTAA) & & 1 & & & 2 & & 1 & 2 & 6 \\
\hline I-box core (GATAA) & 1 & 3 & 3 & 2 & 3 & 1 & 1 & & 14 \\
\hline TCT-motif (TCTTAC) & 2 & & 2 & & & & & 1 & 5 \\
\hline ARE & & 2 & 1 & 1 & 3 & 2 & & 2 & 11 \\
\hline ABRE & 4 & & 1 & & & 1 & & 3 & 9 \\
\hline LTR & & 1 & 1 & & 2 & 1 & 1 & & 6 \\
\hline MBS & 2 & 2 & 2 & 2 & 1 & 2 & 3 & 1 & 15 \\
\hline TCACG-motif & 1 & 1 & 2 & 3 & & 1 & & 2 & 10 \\
\hline TCA-element & 1 & 2 & 3 & 1 & 1 & 3 & 1 & 2 & 14 \\
\hline TC-rich repeats (ATTTTCTTCA) & 1 & 2 & 2 & 2 & 1 & 1 & & & 9 \\
\hline
\end{tabular}

3-AF1 binding site to TCT-motif: light-related cis-elements, ARE to TC-rich repeats: stress-responsive cis-elements, ARE: anaerobic response element, ABRE: ABA-responsive element, LTR: low temperature-responsive element, MBS: MYB binding site, TCACG-motif: MeJA-responsive element, TCA-element: SA-responsive element. The listed cis-elements exist in more than three of the eight gamma-ray-responsive miRNA genes. Numbers represent the number of cis-elements. 
regulatory mechanisms, is necessary for the expression of gamma-ray-specific miRNAs, such as miR840 and miR850. In fact, it was previously reported that miRNA gene promoters are frequently affected by aberrant DNA methylation in human breast cancer, thereby resulting in the decreased expression of miRNAs (Vrba et al., 2013). Therefore, DNA methylation of promoters, or their interaction with active chromatin markers on histones, may also be important for the transcriptional induction of gamma-ray-specific miRNAs.

Table 4. Major predicted cis-elements frequently found in the putative promoter regions of gamma-rayresponsive miRNA genes.

\begin{tabular}{l|c|c|c|c|c|c|c|c|c}
\hline & miR164a & miR169d & miR169h & miR172b* & miR403 & miR827 & miR840 & miR850 & Total \\
\hline AAGAA-motif & 2 & 2 & 2 & 1 & 1 & 1 & & & 9 \\
\hline Box-W1 (TTGACC) & 1 & 1 & 3 & 1 & & 1 & & & 7 \\
\hline CAAT-box & 18 & 43 & 28 & 19 & 35 & 25 & 32 & 23 & 223 \\
\hline CGTCA-motif & 1 & 1 & 2 & 3 & & 1 & & 2 & 10 \\
\hline Skn-1_motif (GTCAT) & 2 & 1 & 3 & 2 & & 2 & 2 & 2 & 14 \\
\hline TATA-box & 76 & 42 & 75 & 94 & 132 & 25 & 15 & 204 & 663 \\
\hline TATCCAT/C-motif & 1 & 2 & & 2 & & 1 & 1 & & 7 \\
\hline Unnamed_4(CTCC) & 5 & 2 & 6 & 7 & 3 & 9 & 13 & 9 & 54 \\
\hline Unnamed_6 (TAAATAT) & 1 & 1 & 1 & 1 & & & & 2 & 6 \\
\hline
\end{tabular}

Listed cis-elements exist in more than five of the eight gamma-ray-responsive miRNA genes. Numbers represent the number of the $c i s$-elements.

\section{Prediction and expression of putative target genes of gamma-ray-responsive miRNAs}

Putative target genes of the gamma-ray-responsive miRNAs were predicted by psRNATarget, a plant small RNA target analysis server (Dai and Zhao, 2011). Target accessions of each miRNA were subjected to post-transcriptional cleavage rather than translational inhibition (Table 5). Twenty-three target accessions were associated with miR164a, while eight accessions were predicted as putative targets for miR840. All target accessions of miR840 are subjected to post-transcriptional cleavage only. However, only limited information is available on the interaction of each miRNA with its target genes. For example, the translation of AGO1 mRNA is inhibited by miR168, which is induced by p19 RNA-silencing suppressor (Várallyay et al., 2010), while miR403 induces the post-transcriptional silencing (cleavage) of the AGO2 gene via interaction with AGO1 (Allen et al., 2005; Harvey et al., 2011). MEMB12 mRNA is targeted by miR393b* in association with AGO2, which is induced by DC3000 (Zhang et al., 2011b). Therefore, we analyzed genome-wide gamma-ray-responsive transcriptomes in order to correlate gamma-ray-responsive miRNAs with the expression of their putative target genes. Only 8 of 121 target accessions (Table 1) were identified in the gamma-rayresponsive transcriptomes with at least a 2-fold change (Table 6). Transcription of At1g70700 or At $1 \mathrm{~g} 30560$ was closely correlated with miR169d in the $c m t 3-11 t$ mutant at 5 days or with miR827 in the wild-type plants 1 day after gamma irradiation, respectively, suggesting that these genes are subjected to miRNA-mediated post-transcriptional control. In contrast, there was only a weak correlation between miR164a and At2g38340, and between miR403 and At3g32110, which can be explained by miRNA-mediated translational inhibition as predicted in Table 5. Moreover, miR403, which showed significantly decreased transcription in the cmt3$11 t$ mutant 1 day after gamma irradiation, did not directly affect the expression of its target gene, At1g31280 (AGO2), in the wild-type and cmt3-11t mutant plants. Increased transcription of the AGO2 gene in the wild-type may be due to its enhanced promoter activity rather than

Genetics and Molecular Research 15 (3): gmr.15038386 
the release of miR403-mediated post-transcriptional silencing, which is induced by pathogen infection in plants. Taken together, the data shown in Tables 1, 5, and 6 suggest that gammaray-responsive miRNAs are rarely associated with their putative target genes in response to gamma rays. Nevertheless, the high gamma-ray-specific inductions of miR840 and miR850 indicate that these miRNAs may affect other target genes unpredictable by psRNATarget.

Table 5. Putative target genes of gamma-ray-responsive miRNAs in Arabidopsis.

\begin{tabular}{|c|c|c|}
\hline miRNA & Inhibition & Target accession \\
\hline \multirow[t]{2}{*}{ miR164a } & Cleavage & $\begin{array}{l}\text { AT1G05890, AT1G31100, AT1G56010, AT1G78240, AT2G17115, AT3G12977, AT3G15170, AT3G26360, } \\
\text { AT3G31935, AT3G33142, AT4G01210, AT4G39400, AT5G07680, AT5G39610, AT5G50770, AT5G53950, } \\
\text { AT5G61430, AT5G65910 }\end{array}$ \\
\hline & Translation & AT1G10530, AT2G37960, AT2G38340, AT4G37705, AT5G19097 \\
\hline \multirow[t]{2}{*}{$\operatorname{miR} 169 \mathrm{~d}$} & Cleavage & $\begin{array}{l}\text { AT1G17590, AT1G48500, AT1G54160, AT1G68560, AT1G70700, AT1G72830, AT2G39210, AT3G05690, } \\
\text { AT3G09915, AT3G20910, AT3G45940, AT4G35080, AT5G06510, AT5G12840, AT5G42120 }\end{array}$ \\
\hline & Translation & AT1G03060, AT1G19810, AT1G80770, AT3G48770, AT4G03795 \\
\hline \multirow[t]{2}{*}{ miR169h } & Cleavage & $\begin{array}{l}\text { AT1G17590, AT1G54160, AT1G68560, AT1G72830, AT3G05690, AT3G20910, AT4G35080, AT5G06510, } \\
\text { AT5G12840, AT5G42120 }\end{array}$ \\
\hline & Translation & AT2G03590, AT2G03600, AT2G10100, AT3G15730, AT3G19540 \\
\hline \multirow[t]{2}{*}{ miR172b* } & Cleavage & $\begin{array}{l}\text { AT1G79730, AT2G03820, AT2G22950, AT2G43465, AT2G47830, AT3G07050, AT3G57330, AT4G10970, } \\
\text { AT4G32420, AT5G48410, AT5G48830, AT5G65720 }\end{array}$ \\
\hline & Translation & AT1G77840, AT2G04110, AT2G16500, AT3G49601, AT3G61820, AT4G12990, AT5G13690 \\
\hline \multirow[t]{2}{*}{ miR403 } & Cleavage & $\begin{array}{l}\text { AT1G03060, AT1G31280, AT1G53210, AT2G01720, AT2G27880, AT3G17310, AT3G17450, AT4G01030, } \\
\text { AT4G08930, AT4G30825, AT5G37110 }\end{array}$ \\
\hline & Translation & AT1G13910, AT1G75820, AT1G77550, AT3G26190, AT3G32110, AT3G49040, AT4G09060 \\
\hline \multirow[t]{2}{*}{ miR827 } & Cleavage & AT1G02860, AT1G30560, AT3G04750, AT4G00610, AT4G08263, AT4G17550, AT4G37590 \\
\hline & Translation & AT1G31760, AT2G10910, AT2G16365, AT2G21450, AT4G01975, AT5G33428, AT5G60615 \\
\hline miR840 & Cleavage & AT1G10580, AT1G50770, AT1G58848, AT1G59218, AT1G64740, AT1G69570, AT2G03150, AT2G26790 \\
\hline \multirow[t]{2}{*}{ miR850 } & Cleavage & $\begin{array}{l}\text { AT1G09340, AT1G41746, AT2G04842, AT2G43240, AT2G44500, AT3G11930, AT3G28007, AT3G42206, } \\
\text { AT3G46630, AT3G50390, AT3G52250 }\end{array}$ \\
\hline & Translation & AT3G59750, AT4G23100, AT4G23470, AT5G16610 \\
\hline
\end{tabular}

Target accessions of eight miRNAs listed in Table 1 were predicted by psRNATarget, a plant small RNA target analysis server, as described by Dai and Zhao (2011).

Table 6. Transcriptional changes of putative target genes of gamma-ray-responsive miRNAs.

\begin{tabular}{|c|c|c|c|c|c|c|}
\hline \multirow[t]{2}{*}{ miRNA } & \multirow[t]{2}{*}{ Target accession } & \multirow[t]{2}{*}{ Target description } & \multicolumn{2}{|c|}{ Wild-type } & \multicolumn{2}{|c|}{ cmt3-11t } \\
\hline & & & Transcription & Correlation & Transcription & Correlation \\
\hline \multirow[t]{3}{*}{ miR164a } & AT2G38340 & DREB-like AP2 domain transcription factor & & & Increase $^{\dagger}$ & Weak \\
\hline & AT5G07680 & NAM (no apical meristem)-like protein; ANAC080 & & & Decrease $^{\dagger}$ & Weak \\
\hline & AT5G39610 & NAM/CUC2-like protein CUC2; ATNAC6 & Decrease $^{\dagger}$ & Weak & & \\
\hline miR169d & AT1G70700 & hypothetical protein; TIFY7 & & & Decrease*; increase $^{\dagger}$ & Weak; strong \\
\hline miR169d, h & AT5G06510 & transcription factor-like protein; NF-YA10 & & & Increase* & Weak \\
\hline \multirow[t]{2}{*}{$\operatorname{miR} 403$} & AT1G31280 & unknown protein; $\mathrm{AGO} 2$ & Increase* & Weak & & \\
\hline & AT3G32110 & non-LTR reverse transcriptase, putative & Increase $^{\dagger}$ & Weak & & \\
\hline $\operatorname{miR} 827$ & AT1G30560 & unknown protein; glycerol-3-phosphate permease homolog & Decrease* & Strong & & \\
\hline
\end{tabular}

*, Transcriptional change of the putative target genes 1 or 5 days after gamma irradiation, respectively. The strength of the correlation was determined by the association between transcriptional changes of the putative target genes and the expression of miRNAs as shown in Table 2. miRNAs and putative target genes with the same change in transcription after gamma irradiation were excluded.

\section{Transcriptional variation of gamma-ray-responsive miRNAs in Arabidopsis wild- type and RNA-directed DNA methylation (RdDM) mutant plants}

Arabidopsis wild-type and cmt3-11t mutant plants undergo significantly different DNA methylation and genome-wide transcriptome changes following exposure to gamma rays (Kim et al., 2013a). Similarly, the profile of gamma-ray-responsive miRNAs differed between the wild-type and cmt3-11t mutant plants (Table 2 and Table S1) and putative target genes of these miRNAs were differentially expressed (Table 6). These results indicate that 
transcriptional changes of miRNA genes in response to gamma rays may be affected by different genotypes such as genome-wide DNA hypomethylation. Moreover, results of our previous study showed that the developmental stage is the main determinant for the profile of gamma-ray-responsive transcriptomes in Arabidopsis (Kim et al., 2013b).

Therefore, we compared the transcription of the eight gamma-ray-responsive miRNAs listed in Table 2 in the wild-type and several RdDM mutants, including such as $c m t 3-11 t$, drm2, $d r d 1-6$, and $d d m 1-2$ using MS medium-grown 14-day-old whole seedlings. In all mutants, transcription of the four gamma-ray-responsive marker genes, At2g30360, At4g19130, At4g22960, and At5g24280, was induced (Kim et al., 2013b; Figure S1). However, most of the miRNAs tested had distinct expression patterns among the wild-type and RdDM mutants, which differed from those observed in the miRNA microarray analysis using soilgrown 28-day-old rosette leaves (Figure 1 and Table 2). Overall expressions of miR164a, miR169d, miR169h, miR172b*, and miR403 were differentially decreased among the wild-
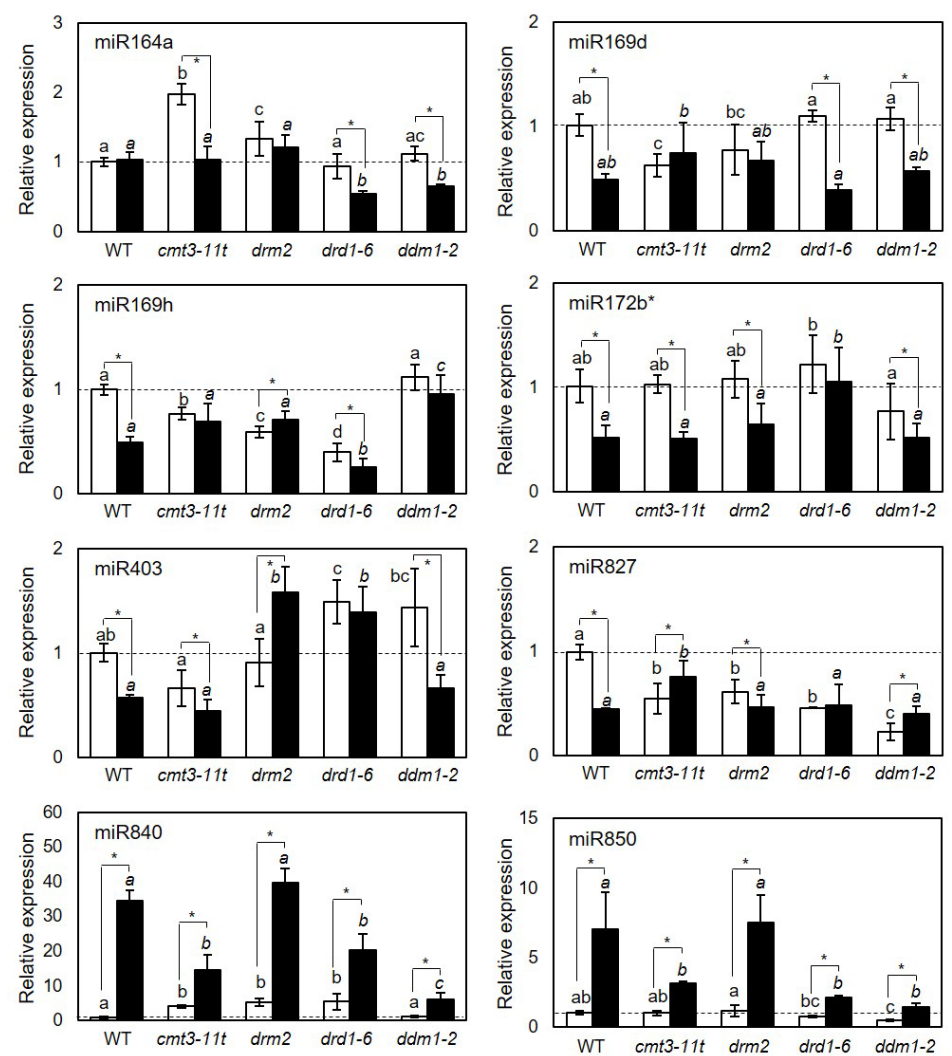

Figure 1. Expression of gamma-ray-responsive miRNAs in Arabidopsis wild-type, cmt3-11t, drm2, drd1-6, and ddm1-2 mutant plants. Total RNA was isolated from MS medium-grown 14-day-old whole seedlings and used for quantitative RT-PCR analysis of each miRNA. All transcript levels are expressed relative to the control samples of the wild-type (WT) plants, as indicated by dashed lines. White and black bars represent means $\pm \mathrm{SD}(\mathrm{N}=6$ from two independent experiments) for the control and irradiated samples, respectively. Bars with the same letters are not significantly different at $\mathrm{P}=0.05$ by the Tukey HSD test. Asterisks indicate statistically significant differences between the control and irradiated samples, as determined by the Student $t$-test $(\mathrm{P}<0.05)$. 
type and mutant plants 1 day after gamma irradiation, while those of miR $169 \mathrm{~h}$ and miR403 were increased specifically in the $d r m 2$ mutant (Figure 1). In contrast to the data presented in Table 2, transcription of miR164a, miR172b*, and miR403 was significantly repressed in the wild-type and in the cmt3-11t mutant. Transcription of miR827, which was strongly induced in the wild-type plants (Table 2), was also found to be repressed by miRNA qRT-PCR analysis using MS medium-grown younger whole seedlings. In contrast, the transcription of miR840 and miR850 was highly induced in the $d r m 2, d r d 1-6$, and $d d m 1-2$ mutants as well as the wild-type and cmt3-11t mutant plants following gamma irradiation. These results indicate that the high transcriptional induction of miR840 and miR850 in response to gamma rays is not significantly affected by the genotype, growth condition, and developmental stage of Arabidopsis plants.

In conclusion, in the present study, investigation of common gamma-ray-responsive miRNAs in Arabidopsis wild-type and various RdDM mutants revealed high transcriptional induction of miR840 and miR850 genes after gamma irradiation. Although the universal radiomiR has yet to be identified in response to ionizing radiation (Joly-Tonetti and Lamartine, 2012), our results suggest that the miRNAs miR 840 and miR850, which are induced by gamma rays, may be reproducible and reliable radiation markers in Arabidopsis. However, further research is needed to elucidate the target genes and functions of these miRNAs in response to gamma rays.

\section{Conflicts of interest}

The authors declare no conflict of interest.

\section{ACKNOWLEDGMENTS}

Research supported by the Nuclear R\&D Program funded by the Ministry of Science, ICT and Future Planning (MSIP), Republic of Korea.

\section{REFERENCES}

Allen E, Xie Z, Gustafson AM and Carrington JC (2005). microRNA-directed phasing during trans-acting siRNA biogenesis in plants. Cell 121: 207-221. http://dx.doi.org/10.1016/j.cell.2005.04.004

Casati P (2013). Analysis of UV-B regulated miRNAs and their targets in maize leaves. Plant Signal. Behav. 8: 4161, 26758. http://dx.doi.org/10.4161/psb.26758

Dai X and Zhao PX (2011). psRNATarget: a plant small RNA target analysis server. Nucleic Acids Res. 39: W155-9. http://dx.doi.org/10.1093/nar/gkr319

Esnault MA, Legue F and Chenal C (2010). Ionizing radiation: Advances in plant response. Environ. Exp. Bot. 68: 231237. http://dx.doi.org/10.1016/j.envexpbot.2010.01.007

Gill SS, Anjum NA, Gill R, Jha M, et al. (2015). DNA damage and repair in plants under ultraviolet and ionizing radiations. ScientificWorldJournal 2015: 250158. http://dx.doi.org/10.1155/2015/250158

Harvey JJW, Lewsey MG, Patel K, Westwood J, et al. (2011). An antiviral defense role of AGO2 in plants. PLoS One 6: e14639. http://dx.doi.org/10.1371/journal.pone.0014639

Jia X, Ren L, Chen QJ, Li R, et al. (2009). UV-B-responsive microRNAs in Populus tremula. J. Plant Physiol. 166: 20462057. http://dx.doi.org/10.1016/j.jplph.2009.06.011

Joly-Tonetti $\mathrm{N}$ and Lamartine $\mathrm{J}$ (2012). The role of microRNAs in the cellular response to ionizing radiations. In: Current topics in ionizing radiation research (Nenoi M, ed.). InTech, Rijeka, 149-174.

Khraiwesh B, Zhu JK and Zhu J (2012). Role of miRNAs and siRNAs in biotic and abiotic stress responses of plants. Biochim. Biophys. Acta 1819: 137-148. http://dx.doi.org/10.1016/j.bbagrm.2011.05.001

Genetics and Molecular Research 15 (3): gmr.15038386 
Kim JE, Lee MH, Cho EJ, Kim JH, et al. (2013a). Characterization of non-CG genomic hypomethylation associated with gamma-ray-induced suppression of CMT3 transcription in Arabidopsis thaliana. Radiat. Res. 180: 638-648. http:// dx.doi.org/10.1667/RR13394.1

Kim J-H, Kim JE, Lee MH, Lee SW, et al. (2013b). Integrated analysis of diverse transcriptomic data from Arabidopsis reveals genetic markers that reliably and reproducibly respond to ionizing radiation. Gene 518: 273-279. http:// dx.doi.org/10.1016/j.gene.2013.01.027

Kruszka K, Pieczynski M, Windels D, Bielewicz D, et al. (2012). Role of microRNAs and other sRNAs of plants in their changing environments. J. Plant Physiol. 169: 1664-1672. http://dx.doi.org/10.1016/j.jplph.2012.03.009

Lescot M, Déhais P, Thijs G, Marchal K, et al. (2002). PlantCARE, a database of plant cis-acting regulatory elements and a portal to tools for in silico analysis of promoter sequences. Nucleic Acids Res. 30: 325-327. http://dx.doi.org/10.1093/ nar/30.1.325

Lhakhang TW and Chaudhry MA (2012). Interactome of radiation-induced microRNA-predicted target genes. Comp. Funct. Genomics 2012: 569731. http://dx.doi.org/10.1155/2012/569731

Li T, Li H, Zhang YX and Liu JY (2011). Identification and analysis of seven $\mathrm{H}_{2} \mathrm{O}_{2}$-responsive miRNAs and 32 new miRNAs in the seedlings of rice (Oryza sativa L. ssp. indica). Nucleic Acids Res. 39: 2821-2833. http://dx.doi. org/10.1093/nar/gkq1047

Liu HH, Tian X, Li YJ, Wu CA, et al. (2008). Microarray-based analysis of stress-regulated microRNAs in Arabidopsis thaliana. RNA 14: 836-843. http://dx.doi.org/10.1261/rna.895308

Moon YR, Kim JH, Lee MH, Kim JS, et al. (2008). Thermal dissipation of excess light in Arabidopsis leaves is inhibited after gamma-irradiation. J. Plant Biol. 51: 52-57. http://dx.doi.org/10.1007/BF03030741

Nagata T, Yamada H, Du Z, Todoriki S, et al. (2005). Microarray analysis of genes that respond to $\gamma$-irradiation in Arabidopsis. J. Agric. Food Chem. 53: 1022-1030. http://dx.doi.org/10.1021/jf0486895

Simone NL, Soule BP, Ly D, Saleh AD, et al. (2009). Ionizing radiation-induced oxidative stress alters miRNA expression. PLoS One 4: e6377. http://dx.doi.org/10.1371/journal.pone.0006377

Várallyay E, Válóczi A, Ágyi A, Burgyán J, et al. (2010). Plant virus-mediated induction of miR168 is associated with repression of ARGONAUTE1 accumulation. EMBO J. 29: 3507-3519. http://dx.doi.org/10.1038/emboj.2010.215

Vrba L, Muñoz-Rodríguez JL, Stampfer MR and Futscher BW (2013). miRNA gene promoters are frequent targets of aberrant DNA methylation in human breast cancer. PLoS One 8: e54398. http://dx.doi.org/10.1371/journal. pone. 0054398

Wang B, Sun YF, Song N, Wang XJ, et al. (2013). Identification of UV-B-induced microRNAs in wheat. Genet. Mol. Res. 12: 4213-4221. http://dx.doi.org/10.4238/2013.October.7.7

Zhang M, Liang S, Hang X, Xiang Y, et al. (2011a). Identification of heavy-ion radiation-induced microRNAs in rice. $A d v$. Space Res. 47: 1054-1061. http://dx.doi.org/10.1016/j.asr.2010.10.024

Zhang X, Zhao H, Gao S, Wang W-C, et al. (2011b). Arabidopsis Argonaute 2 regulates innate immunity via miRNA393(*)mediated silencing of a Golgi-localized SNARE gene, MEMB12. Mol. Cell 42: 356-366. http://dx.doi.org/10.1016/j. molcel.2011.04.010

Zhou X, Wang G and Zhang W (2007). UV-B responsive microRNA genes in Arabidopsis thaliana. Mol. Syst. Biol. 3: 103. http://dx.doi.org/10.1038/msb4100143

\section{Supplementary material}

Table S1. Gamma-ray-responsive miRNAs in Arabidopsis wild-type (WT) or cmt3-11t mutant plants identified by microarray analysis. Ten miRNAs exhibited a $>2$-fold-change in expression 1 day after gamma irradiation in one of three independent experiments. $\dagger$ : 2-fold-change observed in two of three independent experiments.

Figure S1. Expression of gamma-ray-responsive marker genes in Arabidopsis wild-type (WT), cmt3-11t, drm2, drd1-6, and ddm1-2 mutant plants. C and R represent the control and irradiated samples, respectively. ACTIN2 was used as an endogenous control gene. Sense/antisense primer sequences (product size) are as follows: 5'-AgAtGGAGGGGCAAAATGGG-3' / 5'-TGACGACGTCTGATCGCAAA-3' (173 bp) for At2g30360, 5'-TGGAGAAGTGACGACTGAAGC-3' / 5'-ACCTCCAGTTGCGGAACAAT-3' (267 bp) for At4g19130, 5'-AGGGTACAAAAGGGCTCACG-3' / 5'-CTGCAGCATGGTAGCGAAGTGATTGT-3' (167 bp) for At4g22960, 5'-GAGACCGCAGAGCATCTTGA-3' / 5'-GCAAGAGAAGGCATTGGTGC-3' (169 bp) for At5g24280, and 5'-GCCCAGAAGTCTTGTTCCA-3' / 5'-CTTGGTGCAAGTGCTGTGAT-3' (199 bp) for ACTIN2.

Genetics and Molecular Research 15 (3): gmr.15038386 